\title{
Predictors of Distant Metastases in Triple-Negative Breast Cancer Without Pathologic Complete Response After Neoadjuvant Chemotherapy
}

\author{
William R. Kennedy, MDa,*; Christopher Tricarico, BS a,*; Prashant Gabani, MDa; Ashley A. Weiner, MD, PhD; \\ Michael B. Altman, PhDa; Laura L. Ochoa, ARN, PhDa; Maria A. Thomas, MD, PhDa; Julie A. Margenthaler, MD; \\ Souzan Sanati, MD ${ }^{\mathrm{d}}$, Lindsay L. Peterson, MDe; Cynthia X. Ma, MDe; Foluso O. Ademuyiwa, MD; and Imran Zoberi, MD
}

\section{ABSTRACT}

Background: Pathologic complete response ( $\mathrm{pCR}$ ) after neoadjuvant chemotherapy (NAC) for triple-negative breast cancer (TNBC) predicts decreased distant metastasis. However, most patients do not experience $\mathrm{pCR}$, and other risk factors for distant metastasis after NAC are poorly characterized. This study investigated factors predictive of distant metastasis in TNBC without $\mathrm{pCR}$ after NAC. Methods: Women with TNBC treated with NAC, surgery, and radiation therapy in 2000 through 2013 were reviewed. Freedom from distant metastasis (FFDM) was compared between patients with and without pCR using the Kaplan-Meier method. In patients without $\mathrm{PCR}$, univariate and multivariable Cox analyses were used to determine factors predictive of distant metastasis. Results: We identified 153 patients with median follow-up of 4.0 years (range, 0.5-14.0 years). After NAC, 108 had residual disease (pCR, 29\%). Five-year FFDM was $98 \%$ and $55 \%$ in patients with and without $p C R$, respectively $(P<.001)$. Factors independently predicting FFDM in patients without $\mathrm{PCR}$ were pathologic nodal positivity (hazard ratio, $3.08 ; 95 \% \mathrm{Cl}, 1.54-6.14 ; P=.001)$ and lymphovascular space invasion (hazard ratio, $1.91 ; 95 \% \mathrm{Cl}, 1.07-3.43 ; P=.030$ ). Patients with a greater number of factors had worse FFDM; 5-year FFDM was $76.5 \%$ for patients with no factors $(n=38)$ versus $54.9 \%$ and $27.5 \%$ for patients with $1(n=44)$ and 2 factors $(n=26)$, respectively $(P<.001)$. Conclusions: Lack of $\mathrm{pCR}$ after NAC resulted in worse overall survival and FFDM, despite trimodality therapy. In patients with residual disease after NAC, pathologic lymph node positivity and lymphovascular space invasion predicted worse FFDM.

J Natl Compr Canc Netw 2020;18(3):288-296 doi: $10.6004 /$ jnccn.2019.7366

${ }^{a}$ Department of Radiation Oncology, Washington University School of Medicine, St. Louis, Missouri; ${ }^{b}$ Department of Radiation Oncology, University of North Carolina, Chapel Hill, North Carolina; and 'Department of Surgery,

dDepartment of Pathology, and 'Division of Oncology, Washington University School of Medicine, St. Louis, Missouri.

${ }^{*}$ Contributed equally and are co-first authors.

\section{Background}

Triple-negative breast cancer (TNBC) is an often-used surrogate for the basal subtype of breast cancer defined by the lack of estrogen receptor (ER) and progesterone receptor (PR) expression and HER2 gene amplification. As a group, patients with this subtype of breast cancer have a relatively worse prognosis than women with nonTNBC. ${ }^{1}$ This is driven primarily by an increased propensity for distant failure compared with other subtypes of breast cancer, particularly within the first 5 years of diagnosis. ${ }^{2,3}$ Distant failure is a seminal event in the natural history of TNBC, with a 5-year overall survival (OS) rate of approximately $25 \%$ and median survival of 24 months in women with metastatic disease. ${ }^{4}$

With the advent of next-generation sequencing and other technologies, a highly diverse and heterogeneous molecular landscape underlying TNBC has been revealed, and multiple molecular subclassifications have been established. ${ }^{5}$ This heterogeneity is also manifested clinically because TNBC does not respond universally or predictably to current therapies. There is a sharp decrease in breast cancer-specific survival during the first 3 to 5 years after diagnosis with TNBC as opposed to other types of breast cancer, which show a more consistent decline in survival over time. ${ }^{6}$ This is mirrored in their pattern of distant recurrence, in which distant failure is much more common during the first 5 years after diagnosis but then decreases and becomes less common than distant relapse in nonTNBC cancers by 10 years after diagnosis. ${ }^{3}$ Together, this suggests that, although as a whole TNBC is biologically aggressive, there is a subset of patients with curable disease that is sensitive to standard therapy.

Despite the known heterogeneity within TNBC, management has changed little because TNBC is typically still treated as a single disease entity. With the increasing use of neoadjuvant chemotherapy (NAC), one factor that has emerged as an indicator of responsiveness to standard therapy has been the occurrence of a pathologic complete response (pCR) after NAC. There has been some variation in how pCR has been defined in 
the literature, ${ }^{7}$ but is generally defined as a lack of all signs of invasive carcinoma in tissue removed by surgery after treatment with chemotherapy. Patients who exhibit pCR have improved long-term outcomes ${ }^{2}$ with improved disease-free survival (DFS) compared with those who have residual disease. ${ }^{4,8,9}$ However, most women treated with standard anthracycline- and taxane-based NAC do not experience $\mathrm{pCR},{ }^{8,9}$ and data are scarce regarding clinically identifiable prognostic risk factors in this cohort of patients who have residual disease after NAC. We recently identified risk factors for locoregional recurrence (LRR) in patients with TNBC without pCR after NAC. ${ }^{10}$ The present study aimed to identify factors predictive of distant metastasis in patients with TNBC without pCR after NAC.

\section{Methods}

\section{Data Source and Study Population}

An Institutional Review Board-approved database was used as the source for this analysis. All consecutive patients with TNBC treated between 2000 and 2013 with NAC, surgery, and adjuvant radiation therapy (RT) were identified. TNBC was defined by $<1 \%$ ER and PR expression and lack of HER2 expression or gene amplification. Patients with distant metastatic disease at diagnosis or whose disease progressed during NAC were excluded, and no patients with metaplastic breast cancers were included. TNBC was not routinely further subtyped. Patients who did not receive NAC or who experienced disease progression during the course of treatment were also excluded. For the 23 patients (15\%) treated during the 2000 to 2003 period, the specialized breast pathologists at Washington University began testing for HER2 during this time, before approval of adjuvant trastuzumab. Patients who were not classified as having TNBC by virtue of HER2 testing at the time of diagnosis were also excluded.

Patient-, tumor-, and treatment-specific factors were analyzed, including age, race, menopausal status, tumor and node stage, histology, tumor grade, and type of NAC used. Before treatment, all patients were staged clinically with physical examination and diagnostic breast imaging, including mammography and ultrasound. MRI was performed at the discretion of the breast surgeon. PET was not routinely used to clinically stage patients. After NAC, all patients were staged surgically. All surgery was performed at a single institution by specialized breast surgeons, and all specimens were centrally examined at a single institution by a small group of specialized breast pathologists. Standardized protocol for finding the surgical clip was that the clip was grossly searched for by the prosector, and if initial gross examination did not reveal the clip, the specimen was radiographically scanned to locate the clip and examine the tissue accordingly.
The tumor bed was extensively sampled, and in cases with "no residual tumor," the entire tumor bed area was examined before this diagnosis was made. Pathologic downstaging was defined as a decrease in pathologic $\mathrm{T}$ and/or N stage after surgery compared with clinical stage before NAC. pCR was defined as no evidence of invasive disease in the breast or regional lymph nodes (LNs) after NAC, based on hematoxylin-eosin staining routinely. Keratin staining was used occasionally if needed ( $13.7 \%$ of cases). Residual cancer burden scoring was not routinely performed. Multifocal disease was defined as more than one focus of tumor in the same breast at the time of surgery, independent of quadrant or distance. The presence or absence of lymphovascular space invasion (LVSI), extranodal extension, or pathologically positive LNs was determined by a pathologist at the time of surgery. Distant failure was diagnosed clinically, radiographically, and/or pathologically.

\section{Statistical Analysis}

Time intervals were calculated from diagnosis until death or last follow-up. Patients were censored at the date of last clinical contact. OS and freedom from distant metastasis (FFDM) were estimated using the Kaplan-Meier method. The rates of FFDM for patients who experienced pCR and those who did not were compared using log-rank statistics. Univariate and multivariable analyses were performed using the Cox proportional hazards model to evaluate factors associated with distant failure in patients without pCR (ie, those with residual disease) after NAC. To confirm appropriate selection of predictive variables entered into multivariable analysis, a backward logistic regression method (with selection criterion $P<.10$ ) was applied to obtain the final multivariate model. Significance was considered at $P<.05$, and all significance levels were 2 -sided. SPSS Statistics, version 25 (IBM Corp) was applied for all statistical analyses.

\section{Results}

Patients and Treatment Characteristics

A total of 153 patients with a median follow-up of 4.04 years (range, $0.51-13.97$ years) were included in the study cohort. Patient characteristics and treatment strategies are summarized in Table 1. Clinical stage T3 or T4 disease was found in $49 \%$ of patients at diagnosis. Only $25 \%$ of patients were clinically $\mathrm{LN}$-negative at diagnosis, with the remaining $75 \%$ being LN-positive. The most common NAC regimen was AC-T (doxorubicin/cyclophosphamide followed by paclitaxel) $(\mathrm{n}=62 ; 40.5 \%)$. Among all patients, $52.3 \%$ had a mastectomy and $47.7 \%$ underwent partial mastectomy. All patients received adjuvant RT, with most patients $(n=124 ; 81 \%)$ receiving comprehensive $\mathrm{RT}$ to any residual breast tissue, the underlying chest wall, axilla, 


\section{Table 1. Patient- and Treatment-Related} Characteristics

\section{Characteristic}

n (\%)

Total, N

153

Age at diagnosis

$\begin{array}{ll}<50 \mathrm{y} & 78(51) \\ \geq 50 \mathrm{y} & 75(49)\end{array}$

Race

\section{White}

$86(56.2)$

Black or other

$67(43.8)$

Menopausal status

$\begin{array}{ll}\text { Premenopausal } & 78(51)\end{array}$

Postmenopausal

75 (49)

Clinical T stage

\begin{tabular}{ll}
\hline $\mathrm{T} 1$ & $12(7.8)$ \\
\hline $\mathrm{T} 2$ & $66(43.1)$ \\
\hline $\mathrm{T} 3$ & $44(28.8)$ \\
\hline $\mathrm{T} 4$ & $31(20.3)$
\end{tabular}

Clinical $\mathrm{N}$ stage

\begin{tabular}{ll}
$\mathrm{N} 0$ & $38(24.8)$ \\
$\mathrm{N} 1$ & $82(53.6)$ \\
\hline $\mathrm{N} 2-3$ & $33(21.6)$
\end{tabular}

Multifocal disease

\begin{tabular}{lr} 
No & $133(86.9)$ \\
\hline Yes & $20(13.1)$
\end{tabular}

Histology

\begin{tabular}{lr}
\hline Ductal & $135(88.2)$ \\
\hline Other & $18(11.8)$
\end{tabular}

Grade

\begin{tabular}{lr}
\hline I or II & $25(16.3)$ \\
III & $128(83.7)$
\end{tabular}

Pathologic complete response

\begin{tabular}{lr}
\hline No & $108(70.6)$ \\
Yes & $45(29.4)$
\end{tabular}

Pathologic T stage

\begin{tabular}{ll}
\hline T0 & $57(37.3)$ \\
\hline T1 & $44(28.8)$ \\
\hline T2 & $28(18.3)$ \\
\hline T3 & $13(8.5)$ \\
T4 & $11(7.2)$
\end{tabular}

Pathologic N stage

\begin{tabular}{lr}
\hline N0-1 & $125(81.7)$ \\
\hline N2-3 & $28(18.3)$
\end{tabular}

119 (77.8)

Absent

$34(22.2)$

Present

(continued)

\section{Table 1. Patient- and Treatment-Related} Characteristics (cont.)

Characteristic

n (\%)

Margins

Negative $143(93.5)$

Positive

$10(6.5)$

LN assessment

\begin{tabular}{lr}
\hline SLNB & $35(22.9)$ \\
\hline ALND & $118(77.1)$
\end{tabular}

Pathologic LN status

Negative 91 (63.4)

Positive

$62(40.5)$

Extranodal extension

\begin{tabular}{ll}
\hline Absent & $42(66.6)$ \\
\hline Present & $21(33.3)$
\end{tabular}

Downstaging after NAC

No $\quad 52(34.0)$

Yes $101(66.0)$

Surgery type

Partial mastectomy 73 (47.7)

Mastectomy $\quad 80$ (52.3)

NAC type

AC-T 62 (40.5)

FEC-T 27 (17.6)

ET 32 (20.9)

Platinum-based $10(6.5)$

TC $1(0.7)$

Other 21 (13.7)

Adjuvant chemotherapy

No $107(69.9)$

Yes 46 (30.1)

Adjuvant chemotherapy type

ET $22(47.8)$

Platinum-based 9 (19.6)

AC-T $4(8.7)$

Other $11(23.9)$

RT boost

No $52(34.0)$

Yes $101(66.0)$

RT volume

\begin{tabular}{lr} 
RNI & $124(81.0)$ \\
\hline Whole breast & $29(19.0)$
\end{tabular}

Treatment era

2000-2008 $101(66.0)$

2009-2013 $52(34.0)$

Abbreviations: AC-T, doxorubicin/cyclophosphamide/paclitaxel; ALND, axillary lymph node dissection; ET, epirubicin/paclitaxel; FEC-T, fluorouracil/ epirubicin/cyclophosphamide/paclitaxel; LN, lymph node; NAC, neoadjuvant chemotherapy; RNI, regional nodal irradiation; RT, radiation therapy; SLNB, sentinel lymph node biopsy; TC, docetaxel/cyclophosphamide. 
and supraclavicular and internal mammary LNs. The remaining patients received whole-breast RT alone $(\mathrm{n}=29 ; 19.0 \%)$; partial breast RT was not used. The median RT dose was 50.4 Gy (range, 46-60 Gy). A boost was used in $66.0 \%$ of patients, with a median dose of 10 Gy (range, 4-20 Gy). Of patients receiving a boost, $64 \%$ were treated with whole-breast irradiation, and the remaining $36 \%$ received a boost as part of postmastectomy RT. Adjuvant chemotherapy was used in 46 patients $(30.1 \%)$, with ET (epirubicin/docetaxel) being the most common $(n=22 ; 47.8 \%)$, as part of an institutional protocol evaluating 4 cycles neoadjuvant ET followed by 2 cycles of adjuvant ET. ${ }^{11}$ Adjuvant carboplatin was used in 2 patients, and adjuvant capecitabine was used in 1 patient. The most common adjuvant regimen used in patients who received neoadjuvant $\mathrm{AC}-\mathrm{T}$ was platinum-based (5 of 14 patients; $35.7 \%$ ).

\section{Outcomes}

Median OS for the entire cohort was 4.1 years, and the 5 -year OS rate was $67 \%$. For all patients, the overall distant recurrence rate was $31.3 \%$, with a 5-year FFDM of $67 \%$. After NAC, 45 patients $(29.4 \%)$ in our cohort experienced pCR, whereas $108(70.6 \%)$ had residual disease. A larger percentage were at least pathologically downstaged after NAC $(\mathrm{n}=101 ; 66 \%)$. The 5 -year OS rates were $98 \%$ versus $54 \%$ in patients with and without pCR, respectively $(P<.001)$ (Figure $1 \mathrm{~A})$. Distant metastasis occurred in only 1 patient with pCR and 47 patients without pCR. The 5-year FFDM rates were 98\% versus $55 \%$ in patients with pCR and without pCR, respectively $(P<.001)$ (Figure 1B). Among all patients with documented

A

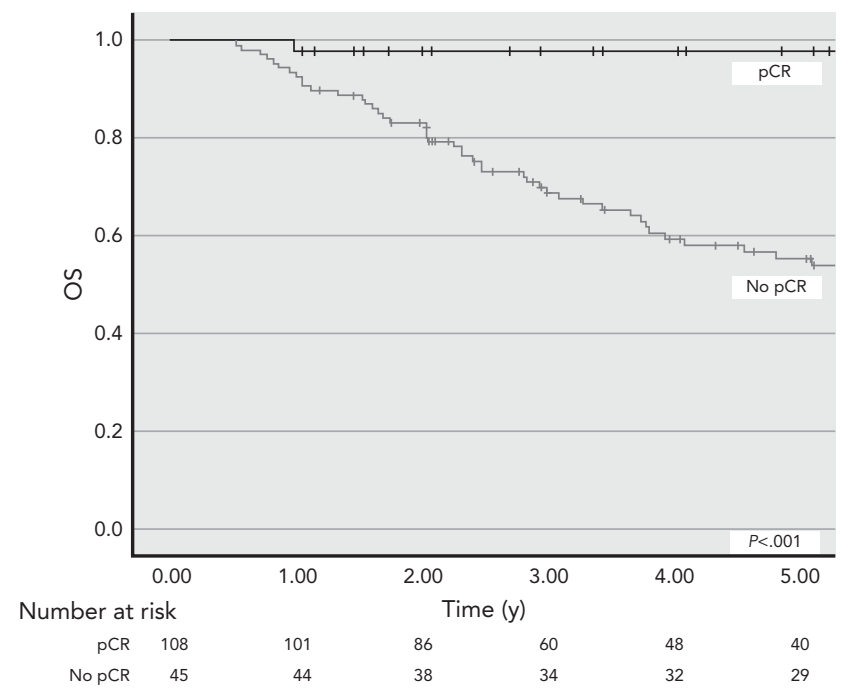

distant metastasis, median time to distant metastasis was 1.25 years (range, $0.34-8.54$ years), and only 5 of 48 patients (10.4\%) had positive margins. Among the 45 patients who experienced pCR, none had an LRR. Among the 108 patients with residual disease after NAC, 21 developed LRR, including 5 in-breast recurrences, 11 nodal recurrences, and 11 chest wall recurrences. Of the nodal recurrences, 8 failed in the axilla, 3 failed in both the axilla and other nodal regions, and no patients had isolated failures in the internal mammary nodes.

\section{Univariate and Multivariable Analyses}

In univariate analysis, factors associated with distant metastasis in the cohort of patients with residual disease after NAC $(n=108)$ included increasing clinical T stage, increasing clinical $\mathrm{N}$ stage, positive pathologic LN status, multifocality, LVSI, extranodal extension, and failure of downstaging after NAC. On multivariable analysis, only positive pathologic LNs (hazard ratio, 3.08; 95\% CI, 1.54-6.14; $P=.001$ ) and LVSI (hazard ratio, 1.91; 95\% CI, $1.07-3.43 ; P=.030$ ) remained significant independent predictors of distant metastasis (Table 2). Figure 2 presents FFDM for patients not experiencing pCR with and without positive pathologic LNs and LVSI. Furthermore, patients with multiple factors had worse FFDM, with a 5-year FFDM of $76.5 \%$ in patients with 0 of these 2 factors $(n=38$ ) versus FFDM rates of $54.9 \%$ and $27.5 \%$ for patients with $1(n=44)$ and 2 factors $(\mathrm{n}=26)$, respectively $(P<.001)$ (Figure 3$)$.

\section{Discussion}

This study identified risk factors predicting distant metastatic disease in patients who do not experience

\section{B}

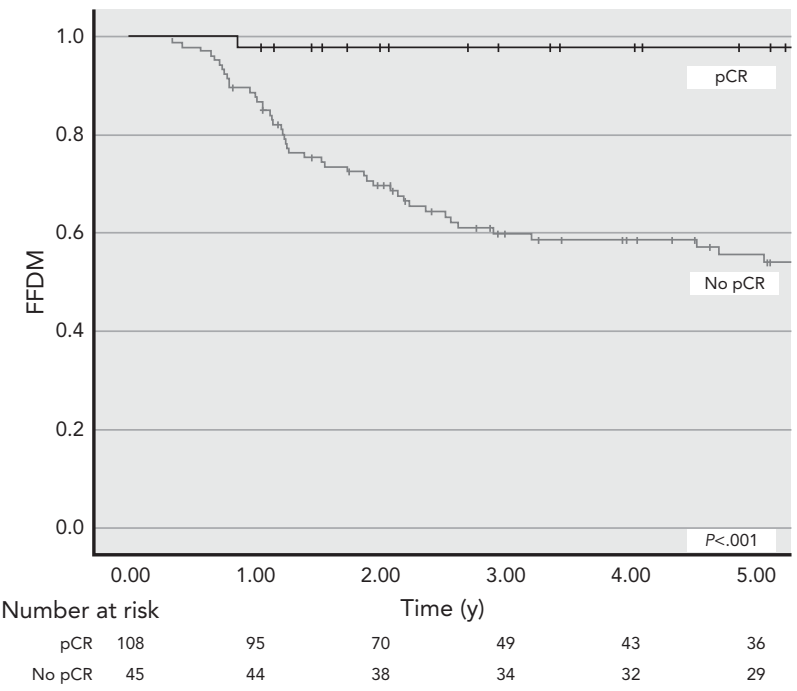

Figure 1. (A) $O S$ and (B) FFDM by $P C R(P<.001$ for both).

Abbreviations: FFDM, freedom from distant metastasis; OS, overall survival; pCR, pathologic complete response. 
Table 2. Univariate and Multivariable Analyses of FFDM in Patients Not Achieving Pathologic Complete Response

\begin{tabular}{|c|c|c|c|c|}
\hline Characteristic & \multicolumn{2}{|c|}{ Univariate Analysis } & \multicolumn{2}{|c|}{ Multivariable Analysis } \\
\hline \multicolumn{5}{|l|}{ Age, y } \\
\hline$<50$ & Ref & & Ref & \\
\hline$\geq 50$ & $1.69(0.95-3.02)$ & .07 & NS & \\
\hline \multicolumn{5}{|l|}{ Race } \\
\hline Black or other & $1.13(0.64-1.99)$ & .67 & NS & \\
\hline \multicolumn{5}{|l|}{ Menopausal status } \\
\hline Postmenopausal & Ref & & Ref & \\
\hline Premenopausal & $0.70(0.40-1.24)$ & .22 & NS & \\
\hline \multicolumn{5}{|l|}{ Clinical T stage } \\
\hline No-1 & Ref & & Ref & \\
\hline N2-3 & $2.06(1.13-3.76)$ & .02 & NS & \\
\hline \multicolumn{5}{|l|}{ Multifocal disease } \\
\hline No & Ref & & Ref & \\
\hline Yes & $2.85(1.48-5.51)$ & .002 & NS & \\
\hline \multicolumn{5}{|l|}{ Histology } \\
\hline Ductal & Ref & & Ref & \\
\hline Other & $1.51(0.71-3.23)$ & .29 & NS & \\
\hline \multicolumn{5}{|l|}{ Grade } \\
\hline Negative & Ref & & Ref & \\
\hline Positive & $1.63(0.65-4.12)$ & .30 & NS & \\
\hline \multicolumn{5}{|l|}{$\mathrm{LN}$ assessment } \\
\hline SLNB & Ref & & Ref & \\
\hline ALND & $2.28(0.97-5.36)$ & .06 & NS & \\
\hline \multicolumn{5}{|l|}{ Pathologic LN status } \\
\hline Negative & Ref & & Ref & \\
\hline Positive & 6.39 (3.32-12.33) & $<.001$ & $3.08(1.54-6.14)$ & .001 \\
\hline \multicolumn{5}{|l|}{ LVSI } \\
\hline Absent & Ref & & Ref & \\
\hline Present & $4.03(2.28-7.12)$ & $<.001$ & $1.91(1.07-3.43)$ & .030 \\
\hline \multicolumn{5}{|l|}{ Extranodal extension } \\
\hline Absent & Ref & & Ref & \\
\hline Present & $5.37(2.97-9.70)$ & $<.001$ & NS & \\
\hline
\end{tabular}




\begin{tabular}{|c|c|c|c|c|}
\hline \multirow[b]{2}{*}{ Characteristic } & \multicolumn{2}{|c|}{ Univariate Analysis } & \multicolumn{2}{|c|}{ Multivariable Analysis } \\
\hline & HR $(95 \% \mathrm{Cl})$ & $P$ Value & $\mathrm{HR}(95 \% \mathrm{Cl})$ & $P$ Value \\
\hline \multicolumn{5}{|l|}{ Downstaging after NAC } \\
\hline No & Ref & & Ref & \\
\hline Yes & $0.29(0.16-0.51)$ & $<.001$ & NS & \\
\hline \multicolumn{5}{|l|}{ Surgery type } \\
\hline Partial mastectomy & Ref & & Ref & \\
\hline Mastectomy & $1.59(0.89-2.83)$ & .12 & NS & \\
\hline \multicolumn{5}{|l|}{ NAC type } \\
\hline$A C-T$ & Ref & & Ref & \\
\hline FEC-T & $1.69(0.77-3.70)$ & .19 & NS & \\
\hline ET & $1.20(0.56-2.57)$ & .64 & NS & \\
\hline Other & $1.22(0.56-2.66)$ & .62 & NS & \\
\hline \multicolumn{5}{|l|}{ Adjuvant chemotherapy } \\
\hline No & Ref & & Ref & \\
\hline Yes & $0.82(0.44-1.52)$ & .52 & NS & \\
\hline \multicolumn{5}{|c|}{ Adjuvant chemotherapy type } \\
\hline Other & Ref & & Ref & \\
\hline Anthracycline-based & $0.69(0.32-1.49)$ & .33 & NS & \\
\hline \multicolumn{5}{|l|}{ RT boost } \\
\hline No & Ref & & Ref & \\
\hline Yes & $1.12(0.61-2.06)$ & .72 & NS & \\
\hline \multicolumn{5}{|l|}{ Treatment era } \\
\hline $2000-2008$ & Ref & & Ref & \\
\hline 2009-2013 & $0.70(0.35-1.38)$ & .30 & NS & \\
\hline
\end{tabular}

Abbreviations: AC-T, doxorubicin/cyclophosphamide/paclitaxel; ALND, axillary lymph node dissection; ET, epirubicin/paclitaxel; FEC-T, fluorouracil/epirubicin/ cyclophosphamide/paclitaxel; FFDM, freedom from distant metastasis; HR, hazard ratio; LN, lymph node; LVSI, lymphovascular space invasion; NAC, neoadjuvant chemotherapy; NS, not significant; RT, radiation therapy; SLNB, sentinel lymph node biopsy.

pCR: pathologic nodal positivity, increasing nodal stage, multifocal disease, and LVSI. Importantly, there was an additive detriment to exhibiting multiple factors, with a 5 -year FFDM rate of $76 \%$ with 0 factors compared with $0 \%$ with all 4 factors. With the aim of identifying appropriate candidates for treatment intensification, our study provides practical foundational work for further risk stratification in patients with TNBC treated with trimodality therapy. Given that these factors are readily available in surgical pathology reports and that they are predictive in patients without pCR, which represents most TNBC cases after NAC, it is our belief that these findings are clinically relevant and applicable in the care of patients with TNBC.

In our cohort, the overall distant recurrence rate was $31.3 \%$, translating to a 5-year FFDM of $67 \%$ for all patients. This is consistent with rates reported in the literature, in which approximately one-third of patients with TNBC experienced a distant recurrence, significantly worse than in non-TNBCs, with distant metastasis rates of approximately $20 \% .^{3}$ Similarly, Haffty et al ${ }^{12}$ showed a comparable distant metastasis rate of $29 \%$ at 5 years in their analysis of 117 patients with TNBC compared with $12 \%$ among patients without TNBC. In addition, the pCR rate after NAC in our cohort was $29.4 \%$, which is also consistent with data in the TNBC literature that typically range from $30 \%$ to $40 \% .^{8}$

Despite the known heterogeneity underlying TNBC, rates of distant metastasis reported in the literature are relatively consistent. In contrast, the rate of isolated LRR in TNBC has varied in the literature from $4.6 \%$ to $22 \% .{ }^{12,13}$ Taken together, this supports the notion that there may be a regularly occurring and potentially definable subset of TNBC with resistance to standard chemotherapies. This subset may be responsible for lack of pCR and, ultimately, the consistently observed frequency of distant relapse across populations and treatment modalities. 
A

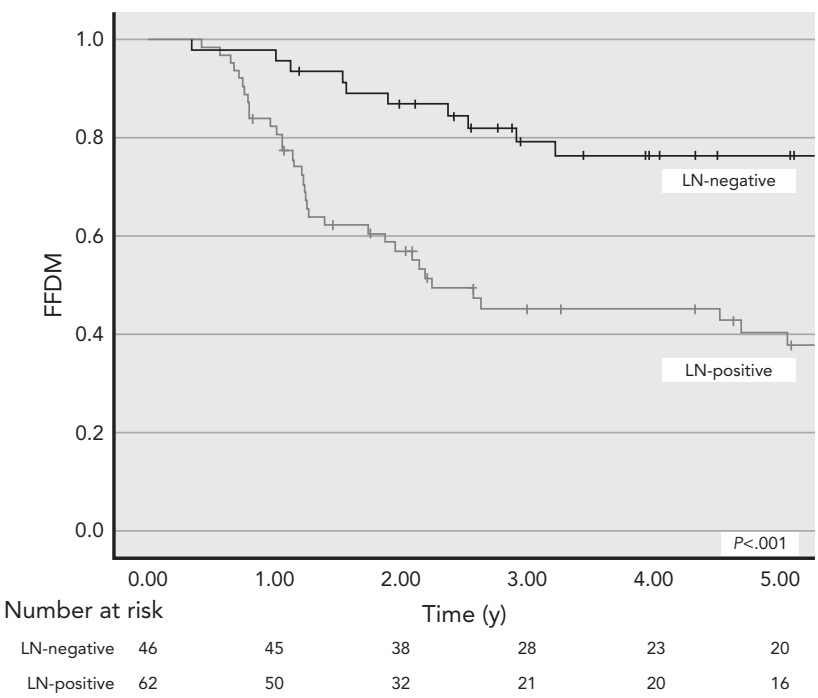

B

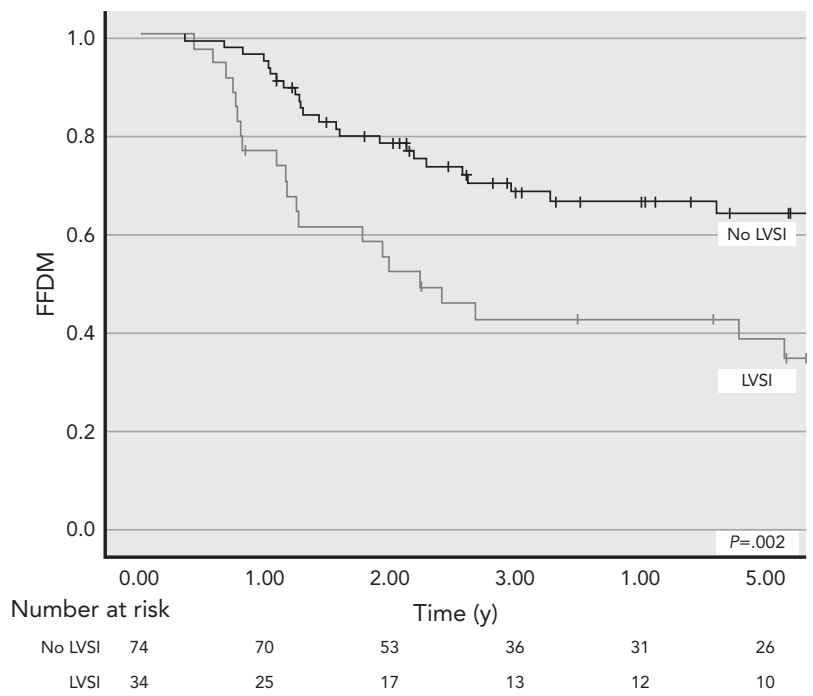

Figure 2. FFDM by $(A)$ pathologic $L N$ status $(P<.001)$ and $(B) \operatorname{LVSI}(P=.002)$ in patients without pathologic complete response after neoadjuvant chemotherapy.

Abbreviations: FFDM, freedom from distant metastasis; LN, lymph node; LVSI, lymphovascular space invasion.

It has been reported that the risk of distant recurrence in patients with TNBC is highest within the first 3 to 5 years after diagnosis and treatment, as opposed to in those without TNBC, who show more consistent distant relapse rates over time. ${ }^{1,3}$ These findings are corroborated by our results, which showed a median time to distant metastasis of 1.25 years, with $94 \%$ of the distant recurrences

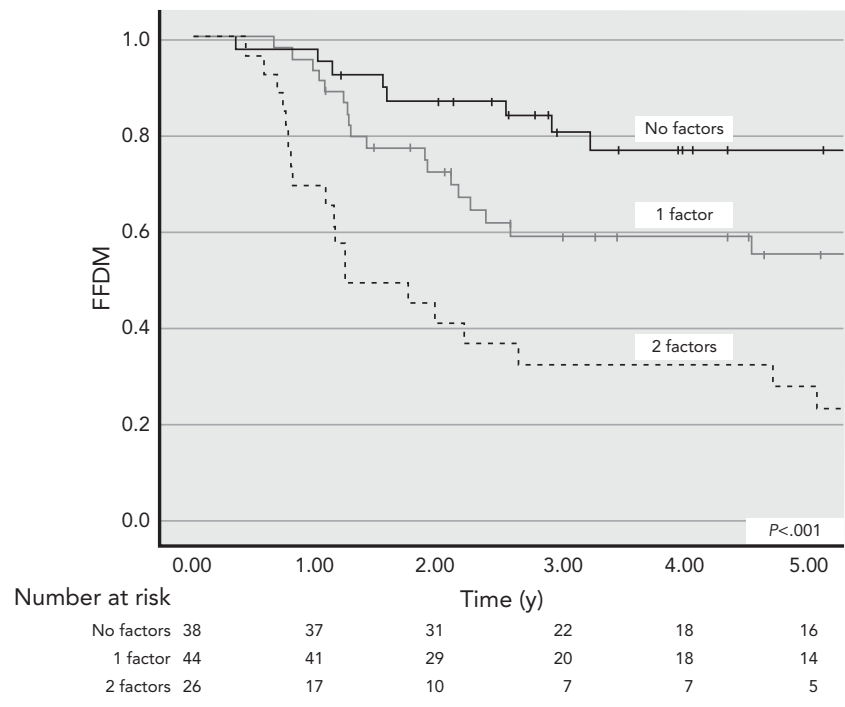

Figure 3. FFDM in patients without pathologic complete response, stratified by number of factors significant in multivariable analysis, including pathologic node positivity and lymphovascular space invasion $(P<.001)$.

Abbreviation: FFDM, freedom from distant metastasis. occurring by 5 years, further supporting the idea that although biologically aggressive as a group, many TNBCs are potentially curable. Our multivariable analysis revealed that positive LNs and increasing pathologic N stage (defined as pN2-3 disease) were both associated with distant metastasis. This result is consistent with other analyses showing that nodal positivity and increasing pathologic $\mathrm{N}$ stage are associated with worse OS and FFDM. ${ }^{14-17}$ This may be explained by the hypothesis that patients with early LN involvement may already have radiographically occult distant metastasis, which is a subsequent driver of mortality in TNBC. ${ }^{18}$

LVSI is more commonly observed in patients with TNBC than in those without TNBC. ${ }^{19}$ Ahn et al ${ }^{20}$ also found LVSI to be a poor prognostic factor for both DFS and OS in both univariate and multivariate analyses. However, not all studies have shown LVSI to influence survival. Urru et $\mathrm{al}^{21}$ found that LVSI was not significantly associated with survival in multivariate analysis. Regardless of the implications for survival, several groups have shown that the presence of LVSI predicts the development of distant metastatic disease. ${ }^{21-23}$ Therefore, our results showing LVSI to be independently predictive of distant metastasis in patients without pCR are in accordance with the literature.

Multifocality is defined as $\geq 2$ tumors in the same quadrant of the breast. Complex literature has been published on this topic, although to date multifocality has been primarily examined indiscriminately in the 
context of all breast cancers, with most studies not reporting subgroup data for patients with TNBC. Some groups have reported a greater probability of nodal metastases and relapse and worse survival in multifocal tumors, but these associations have been inconsistent, with other groups showing that multifocality is not an independent predictor of prognosis in multivariate analysis. ${ }^{24-26}$ Although significant in univariate analysis, multifocality did not remain independently predictive of distant metastasis in our study. Of particular interest to the study of TNBC, between $4.4 \%$ and $15.9 \%$ of foci have been noted to be discordant in their receptor expression. ${ }^{27} \mathrm{~A}$ recent meta-analysis showed that multifocality is associated with significantly worse OS and strong trends toward worse DFS compared with unifocal tumors. ${ }^{28}$ Furthermore, adverse outcomes have been observed in patients with multifocal tumors specifically after NAC. ${ }^{25,29}$ Despite this, the true impact of multifocality is still somewhat controversial because of the heterogeneity of studies and the presence of other confounding factors, including the observations that multifocal tumors are more likely to receive aggressive therapy and that multicentric tumors are often included in the group of multifocal tumors in some studies. It is our institutional practice to define multifocal tumors as those with $>1$ focus in the same breast, regardless of whether they are in the same quadrant or of the distance between foci. Multicentric tumors are defined, both traditionally and at our institution, as those with multiple foci of disease in separate quadrants. This more comprehensive criterion used in our study therefore includes what are traditionally defined as both multifocal and multicentric tumors.

The challenge remains to identify new treatment paradigms for the $60 \%$ to $70 \%$ of patients who do not experience pCR after NAC. Although improvements in adjuvant therapy, such as capecitabine, have improved outcomes in these patients, those with TNBC still have inferior OS and DFS compared with their non-TNBC counterparts. ${ }^{30}$ Next-generation sequencing and RNA expression analysis have identified targetable alterations in pathways, with agents currently under investigation in as many as $90 \%$ of patients with TNBC who do not experience pCR after NAC according to sampling of the residual chemoresistant portion of the tumor. ${ }^{31}$ As the cost of next-generation sequencing technologies continues to decrease, patients not experiencing $\mathrm{pCR}$, and in particular those with high-risk features such as nodal status and LVSI, will be prime candidates for further sequencing studies and use of more targeted systemic therapies. ${ }^{32}$ Intuitively, targeting of the pathways in the residual tumor persistent after NAC may also treat micrometastatic disease ultimately responsible for distant metastasis, because these pathways would be derived from this pool of persistent chemoresistant tumor cells. ${ }^{31}$

Further challenges also remain because of the issue of clonal evolution among these tumors. Genomic analyses have revealed that many TNBCs show substantial clonality and intratumoral heterogeneity, consistent with their association with homologous recombination (HR) deficiency and intrinsic genomic instability. On average, there are approximately 60 somatic coding mutations per tumor, with some harboring as many as 160 per tumor. ${ }^{5}$ This has important clinical implications for the development of resistance to therapies and lack of response to targeted therapies. For example, if there is an initiating mutation in the HR pathway, this would confer sensitivity to standard DNAdamaging therapy, as described earlier; however, there could be development of a mutation in a subclone that reverses HR deficiency and reverses the sensitivity to DNA-damaging agents, which could then survive treatment and cause relapse. As an example, preclinical data show that loss of PTEN can reverse HR deficiency in BRCA1-deficient cells and reverse the added sensitivity to DNA-damaging agents. This is relevant because loss of $P T E N$ is recurrently mutated in up to $9.6 \%$ of TNBCs, and comutations of BRCAI and PTEN are frequent. ${ }^{33}$ This highlights the need to develop effective strategies for assessing intratumoral heterogeneity and clonal evolution in this particularly genetically unstable group of tumors.

Our study had several limitations. We included patients with TNBC who were diagnosed based on standard biomarker analysis of ER, PR, and HER2 via immunohistochemistry. Gene expression profiling has resulted in the classification of breast cancer into 5 intrinsic subtypes, of which basal-like breast cancer most closely overlaps with TNBC. Approximately $80 \%$ of TNBCs as identified by immunohistochemistry are basal-like breast cancers by gene expression profiling; however, these categories are not synonymous, and gene expression profiling remains the gold standard for identification of this clinical entity. ${ }^{1,30}$ Thus, differences in intrinsic TNBC subtypes may affect these observed differences. As a retrospective study, our analysis was also limited by selection bias. The study cohort was derived from a single institution with a tertiary referral pattern, and our patient population may not accurately represent the patterns of care at other institutions. The adjustment variables used in our multivariable analysis may have been incomplete, and some variables for which data were not collected include LN positivity ratio, Ki67, tumor-infiltrating lymphocytes, p53 status, and necrosis, the absence of which could affect our results. 


\section{Conclusions}

Our study highlights the established poor prognosis of patients with TNBC who do not experience pCR after NAC. Furthermore, our findings show that among this cohort of women, there is still significant heterogeneity in outcomes. We have identified positive LNs and LVSI as significant risk factors predicting distant metastatic disease among patients without pCR. These data can be used to stratify patients in relation to their prognoses and potentially inform treatment decisions among these high-risk patients. Prospectively designed trials are warranted to investigate novel therapeutic approaches aimed at improving the high rates of distant failure and relatively poor survival in this high-risk cohort.

Submitted April 10, 2019; accepted for October 3, 2019.

Author contributions: All authors listed contributed significantly to warrant authorship of the present work.

Disclosures: The authors have disclosed that they have not received any financial consideration from any person or organization to support the preparation, analysis, results, or discussion of this article.

Correspondence: Imran Zoberi, MD, Department of Radiation Oncology, Center for Advanced Medicine, Washington University School of Medicine, 4921 Parkview Place, Lower Level, St. Louis, MO 63110. Email: izoberi@wustl.edu

\section{References}

1. Foulkes WD, Smith IE, Reis-Filho JS. Triple-negative breast cancer. N Engl J Med 2010;363:1938-1948.

2. Liedtke C, Mazouni C, Hess KR, et al. Response to neoadjuvant therapy and long-term survival in patients with triple-negative breast cancer. J Clin Oncol 2008;26:1275-1281.

3. Dent R, Trudeau M, Pritchard KI, et al. Triple-negative breast cancer: clinical features and patterns of recurrence. Clin Cancer Res 2007;13: 4429-4434.

4. Bonotto M, Gerratana L, Poletto E, et al. Measures of outcome in metastatic breast cancer: insights from a real-world scenario. Oncologist 2014;19:608-615.

5. Bianchini G, Balko JM, Mayer IA, et al. Triple-negative breast cancer: challenges and opportunities of a heterogeneous disease. Nat Rev Clin Oncol 2016;13:674-690.

6. Cheang MC, Voduc D, Bajdik C, et al. Basal-like breast cancer defined by five biomarkers has superior prognostic value than triple-negative phenotype. Clin Cancer Res 2008;14:1368-1376.

7. Kuroi K, Toi M, Ohno S, et al. Comparison of different definitions of pathologic complete response in operable breast cancer: a pooled analysis of three prospective neoadjuvant studies of JBCRG. Breast Cancer 2015;22:586-595.

8. von Minckwitz G, Untch M, Blohmer JU, et al. Definition and impact of pathologic complete response on prognosis after neoadjuvant chemotherapy in various intrinsic breast cancer subtypes. J Clin Oncol 2012;30: 1796-1804.

9. Cortazar P, Zhang L, Untch M, et al. Pathological complete response and long-term clinical benefit in breast cancer: the CTNeoBC pooled analysis. Lancet 2014;384:164-172.

10. Gabani P, Merfeld E, Srivastava AJ, et al. Predictors of locoregional recurrence after failure to achieve pathologic complete response to neoadjuvant chemotherapy in triple-negative breast cancer. J Natl Compr Canc Netw 2019;17:348-356.

11. Aft R, Naughton M, Trinkaus $K$, et al. Effect of zoledronic acid on disseminated tumour cells in women with locally advanced breast cancer: an open label, randomised, phase 2 trial. Lancet Oncol 2010;11:421-428.

12. Haffty $B G$, Yang $Q$, Reiss $M$, et al. Locoregional relapse and distant metastasis in conservatively managed triple negative early-stage breast cancer. J Clin Oncol 2006;24:5652-5657.

13. Steward L, Conant $L, G a o F$, et al. Predictive factors and patterns of recurrence in patients with triple negative breast cancer. Ann Surg Oncol 2014;21:2165-2171.

14. Hernandez-Aya LF, Chavez-Macgregor M, Lei X, et al. Nodal status and clinical outcomes in a large cohort of patients with triple-negative breast cancer. J Clin Oncol 2011;29:2628-2634.

15. Chen $H$, Ding $A$, Wang $M$, et al. Prognostic significance of lymph node metastasis in triple negative ductal carcinoma of the breast: a retrospective cohort study. Int J Clin Exp Med 2017;10:2727-2736.

16. Dings PJ, Elferink MA, Strobbe LJ, et al. The prognostic value of lymph node ratio in node-positive breast cancer: a Dutch nationwide populationbased study. Ann Surg Oncol 2013;20:2607-2614.
17. Shen ZZ. Relation of tumor size, lymph node status and prognosis in breast cancer [in Chinese]. Zhonghua Wai Ke Za Zhi 1991;29:554-557, 589.

18. Liu HT, Ma R, Yang QF, et al. Lymphangiogenic characteristics of triple negativity in node-negative breast cancer. Int J Surg Pathol 2009;17: 426-431.

19. Agarwal G, Nanda G, Lal P, et al. Outcomes of triple-negative breast cancers (TNBC) compared with non-TNBC: does the survival vary for all stages? World J Surg 2016;40:1362-1372.

20. Ahn KJ, Park J, Choi Y. Lymphovascular invasion as a negative prognostic factor for triple-negative breast cancer after surgery. Radiat Oncol J 2017; 35:332-339.

21. Urru SAM, Gallus S, Bosetti C, et al. Clinical and pathological factors influencing survival in a large cohort of triple-negative breast cancer patients. BMC Cancer 2018;18:56.

22. Rakha EA, Martin S, Lee AHS, et al. The prognostic significance of lymphovascular invasion in invasive breast carcinoma. Cancer 2012;118: 3670-3680.

23. Mohammed RA, Martin SG, Gill MS, et al. Improved methods of detection of lymphovascular invasion demonstrate that it is the predominant method of vascular invasion in breast cancer and has important clinical consequences. Am J Surg Pathol 2007;31:1825-1833.

24. Neri A, Marrelli D, Megha T, et al. Clinical significance of multifocal and multicentric breast cancers and choice of surgical treatment: a retrospective study on a series of 1158 cases. BMC Surg 2015;15:1.

25. Ataseven B, Lederer B, Blohmer JU, et al. Impact of multifocal or multicentric disease on surgery and locoregional, distant and overall survival of 6,134 breast cancer patients treated with neoadjuvant chemotherapy. Ann Surg Oncol 2015;22:1118-1127.

26. Winters ZE, Horsnell J, Elvers KT, et al. Systematic review of the impact of breast-conserving surgery on cancer outcomes of multiple ipsilateral breast cancers. BJS Open 2018;2:162-174.

27. Buggi F, Folli S, Curcio A, et al. Multicentric/multifocal breast cancer with a single histotype: is the biological characterization of all individual foci justified? Ann Oncol 2012;23:2042-2046.

28. Vera-Badillo FE, Napoleone M, Ocana A, et al. Effect of multifocality and multicentricity on outcome in early stage breast cancer: a systematic review and meta-analysis. Breast Cancer Res Treat 2014;146:235-244.

29. Moon HG, Han W, Kim JY, et al. Effect of multiple invasive foci on breast cancer outcomes according to the molecular subtypes: a report from the Korean Breast Cancer Society. Ann Oncol 2013;24:2298-2304.

30. Masuda N, Lee SJ, Ohtani S, et al. Adjuvant capecitabine for breast cancer after preoperative chemotherapy. N Engl J Med 2017;376:2147-2159.

31. Balko JM, Giltnane JM, Wang K, et al. Molecular profiling of the residual disease of triple-negative breast cancers after neoadjuvant chemotherapy identifies actionable therapeutic targets. Cancer Discov 2014;4:232-245.

32. Pareek CS, Smoczynski R, Tretyn A. Sequencing technologies and genome sequencing. J Appl Genet 2011;52:413-435.

33. Peng G, Chun-Jen Lin C, Mo W, et al. Genome-wide transcriptome profiling of homologous recombination DNA repair. Nat Commun 2014;5: 3361. 